\title{
Robot-Assisted Endoscopic Surgery
}

National Cancer Institute

\section{Source}

National Cancer Institute. Robot-Assisted Endoscopic Surgery. NCI Thesaurus. Code C116508.

A surgical technique that uses a specially designed robotic system to aid with an endoscopic procedure. 